\title{
Directed Cultural Probes: Detecting Barriers in the Usage of Public Transportation
}

\author{
Susanne Schmehl ${ }^{1}$, Stephanie Deutsch ${ }^{1}$, Johann Schrammel ${ }^{1}$, Lucas Paletta ${ }^{3}$, \\ and Manfred Tscheligi ${ }^{1,2}$ \\ ${ }^{1}$ CURE -Center for Usability Research and Engineering, Modecenterstr.17/2 \\ 1110 Vienna, Austria \\ \{schmehl, deutsch, schrammel, tscheligi\} acure.at \\ ${ }^{2}$ ICT\&S Center, University of Salzburg, Sigmund-Haffner-Gasse 18 \\ 5020 Salzburg, Austria \\ manfred.tscheligi@sbg.ac.at \\ ${ }^{3}$ JOANNEUM RESEARCH ForschungsgesmbH, Wastiangasse 6, \\ 8010 Graz, Austria \\ lucas.paletta@joanneum.at
}

\begin{abstract}
In this paper we describe the application of a variation of cultural probing for identifying barriers in the use of public transportation for target groups with visual, cognitive or language-related handicaps. To be able to better focus on the targeted aspect - the barriers - we applied modifications to the traditional cultural probing approach: Users were encouraged to generate data related to the targeted aspect. We found that this approach can produce focused results that can be analysed fast and can help to overcome obstacles related to limitations in verbal skills or expressiveness of the user.
\end{abstract}

Keywords: User requirements, Cultural Probes, Directed Cultural Probes, elderly, immigrants, functional illiterates, public transportation.

\section{Introduction}

The good understanding of the users' view of the world and their needs is an essential prerequisite for designing usable products and tools. To improve quality and gathering of such information, different methodological approaches are continuously elaborated. Within HCI a qualitative method called cultural probing, has been introduced, which is used to gain insights into users living context and behaviour aspects in given contexts (such as the use of certain technology). Cultural probing benefits from enhanced user involvement, as participants are engaged to autonomously capture personal impressions from their daily life. Originally introduced by Gaver and colleagues [1], cultural probes encourage participants to generate materials in everyday situations by use of different means (e.g. camera, diary). Hence, the method allows participants to note subjective impressions using textual and graphical illustrations apart from restricted questionnaire formats. The main benefit of the concept is the richness of the user-generated data based on subjective perceptions. Whereas this approach serves to collect large amounts of 
diverse information from a specific context, results from this highly subjective data need to be interpreted qualitatively. During analysis interpreters usually seek to deduce all-encompassing evidence from heterogeneous data. Similarly, they are required to process their data in ways that prevent from informational loss.

In this paper we describe the realization of a study using modified cultural probing for the identification of subjective barriers that people with special needs encounter in public transportation facilities. We report advantages and shortcomings of the new approach as well as we discuss the application possibilities and whether the method is tailored appropriately to the abilities of three user groups.

\section{Related Work}

Since Gaver and colleagues started doing research with cultural probes, many variations have been elaborated. Most of these variations contain diaries and photography in some form. Diaries are used to capture activities in context, to understand needs and motivations related to the use of a certain technology and to gather user requirements for design [2]. The application of the method gradually developed from an inspirational to an informal function. By conducting Technology Probes [3] existing technologies can be situated in users' homes to inspire design by exposing users to new experience. Empathy Probes [e.g. 4] have been designed to see and understand people's emotions and feelings in their natural environment, in order to support design processes. Mattelmäki and colleagues included a small diary booklet, a sheet of stickers, a disposable camera with a list of photography assignments, and ten illustrated cards with open questions. Hulkko and colleagues [5] developed a new digital contextual and self-documenting tool for studying people's actions in mobile contexts. They used mobile phones with GPRS connections, an external digital camera and a developed system for sharing and sorting the data. Crabtree and colleagues [6] proposed their Informal Probes employing various biographical approaches to encourage participants to reflect upon and articulate important personal, social, and technological features of their everyday lives. These reflections enabled designers as well as participants to formulate and elaborate the role of design in the studied culture. Until today, many variations of Gaver's cultural probes have been deployed.

Nevertheless, the richness of the collected data might also be a disadvantage in concerns of analysing the data. The traditional approach of cultural probing typically produces a huge amount of unstructured qualitative data. Additionally, it is difficult and time-consuming to identify the relevant material within this plenitude of created data. In order to differentiate from the original purpose of Gaver's concept [1] - to inspire design - and from the solely identification of user needs, we developed a variation, which asks the user to actively focus on specific problems and to detect and document relevant situations as well as to provide suggestions of how to solve the problems. We used this approach to directly detect barriers in public transportation for people with special needs. 


\section{Directed Cultural Probes}

For the study a modified, directed cultural probing (DCP) method was elaborated. Instead of documenting the whole contextual experience, the documentation was explicitly directed to a restricted issue of the context, focused on barriers in the use of public transportation facilities. The participants - a group of elderly, a group of functional illiterates, and a group of immigrants - had to follow a standardised documentation scheme with the goal to produce focused data. The challenge was to gather and compare qualitative data from different user groups using different materials. Since participants differed in reading and language skills, the DCPs had to be adapted to their special abilities thoroughly. Hence, we will show how this approach is also suitable for contextual inquiries with other user groups.

\subsection{Development}

When compiling the probing packages, special requirements in relation to participants' ability to understand textual information were taken into account. In a first step a workshop with representatives of the end user organisations of three social groups was conducted on how to design the cultural probing packages in respect to specific end user needs. A generic manual served as a basis to discuss on suitability of the probes for the groups. For sending the elderly persons in the field, it was crucial that they did not have to write down things immediately. According to their physical condition it was argued that note taking in a sitting position would be more comfortable. A diary (instead of a voice recorder) was recommended in paper-pencil form to write down their experiences for elderly people and immigrants. The immigrants were allowed to complete the diary in their mother tongue, otherwise the additional effort for the participants might have kept them from documenting properly and some essential information might have gone lost.

Table 1. Different composition of the probing packages per end user group

\begin{tabular}{lccc}
\hline & Elderly & Illiterates & Immigrants \\
\hline Probing package & $\begin{array}{c}\text { diary at home, } \\
\text { camera, checklist }\end{array}$ & $\begin{array}{c}\text { voice recorder, } \\
\text { camera, checklist }\end{array}$ & $\begin{array}{c}\text { diary, camera, } \\
\text { checklist }\end{array}$ \\
\hline
\end{tabular}

Finally, workshop participants agreed that participants of all groups could document their experienced barriers by using a disposable camera (max. 27 pictures). The participants were asked to take a picture of the detected barrier and to comment as soon as possible on the required entries. However, according to the participants all diary entries (written or spoken) were made on the same day as the picture was taken.

Instead of the diary, the functional illiterates received a digital voice recorder (Olympus VN-5500) to comment the detected barriers right after taking the picture. They had a checklist on their voice recorder. To produce structured data each photograph had to be commented by a strict documentation scheme. The scheme also served as the checklist provided to all participants: date and time, name and brief description of the barrier, name of leg of the journey, emotional state in relation to the 
barrier (5-smiley scale), behavioural reactions of the surrounding, ideas or wishes to eliminate or minimize the barrier.

The checklist was adapted for user groups: a translated version for immigrants and a version with extra-large letters for the elderly and the functional illiterates. Illustrations with pictures depicting the legs of journey should serve a better understanding by all user groups.

\subsection{Participants}

Three user groups were involved in the investigation: elderly people with visual and cognitive impairments, immigrants with initial lack of expertise about local language and functional illiterates with principal problems in the understanding of text. The elderly group consisted of 10 persons ( 4 men, 6 women). Their impairments varied in terms of degree of vision (e.g. nearly blind) and physical mobility (e.g. walking aid). During recruiting special prerequisites were defined: their ability to use public transportation independently, to reflect their loss of memory (in relation to dementia), and to be in a stable health condition, even if being under medication. 12 functional illiterates ( 7 men, 5 women) participated. Their mother tongue had to be German and their linguistic competency levels had to vary. The degree of variation was determined by the end user organisation. 11 immigrants ( 1 male, 10 women $\left.{ }^{1}\right)$ took part in the study. At recruiting they had to establish the following preconditions: maximum duration of stay in Austria of 6 years and relatively bad German language skills. A high diversity of languages within the group of immigrants was striven for (8 different languages), to cover as much language diversity as possible.

Table 2. Total number of participants and their age (at the beginning of data acquisition)

\begin{tabular}{lccc}
\hline & Elderly & Illiterates & Immigrants \\
\hline $\begin{array}{l}\text { number of participants } \\
\text { average age of participants } \\
\text { (mean } \pm \text { stdv) }\end{array}$ & 10 & 12 & 11 \\
\hline
\end{tabular}

All participants used public transport previously. For the duration of the study, they were requested to use public transportation as often as possible, to ensure that enough data was collected. However, in order to avoid the generation of pseudo-barriers no minimum or maximum of barriers to identify was requested from participants.

\subsection{Study Procedure}

For the study the DCP method was combined with pre-interviews and post-focus groups. The way of how barriers were documented varied between the groups according to their special needs. After acquisition, data was reprocessed and focus

\footnotetext{
${ }^{1}$ Most women neither had a car nor a driving license, that's why women rather than men use public transportation. Consequently, this skewed sex ratio within the immigrant group reflects realistic demographic conditions.
} 
groups were conducted to discuss and prioritise the detected barriers by the end user themselves.

Pre-Interview. Participants were pre-interviewed in order to get first insights: in their demography and daily habits (e.g. use of public transportation), as well as in their social environment (e.g. family situation). Furthermore, participants were asked to report on perceptions and problematic situations they previously have been confronted with in public transportation, as well as on technology use and acceptance (e.g. cell phone, internet usage). The questions of the pre-interviews varied slightly in detail for the three groups, but roughly stayed within the mentioned domains. For the pre-interviews with the immigrants an interpreter was present. After the preinterview, participants received their personal DCP package. They were instructed to actively look for barriers that occur for them personally while using public transportation and further to document them. Informational background was provided, that results would be used for the conception of a mobile assistance service.

Probing. Over two weeks participants had to find as many barriers as possible by traveling through the city for their daily routines. The data acquisition took place in August 2010. Due to different personal reasons of the participants (e.g. illness) the number of active participants decreased during the data collection. After the data collection probing packages of only 9 elderly, 11 illiterates and 7 immigrants were delivered.
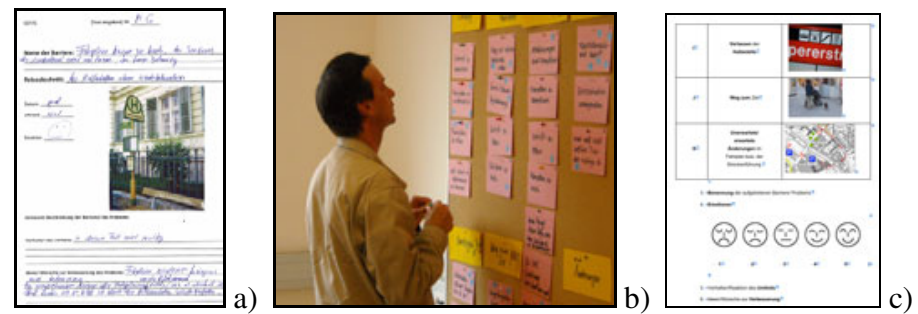

Fig. 1. a) Reprocessing in the diary, b) post focus group participant prioritising barriers, c) extract from the checksheet

Reprocessing. After two weeks of data acquisition participants returned the disposable cameras as well as the textual or oral diaries to their end user organisations. After photographs had been developed, participants glued each photograph to the corresponding diary entry (Fig. 1a) with assistance of co-workers from the end user organisations. As the immigrants were allowed to write diary entries in their mother tongue, an interpreter supported the elaboration of the immigrants' probes.

Focus Groups. In three separate focus groups participants presented their reprocessed data sheets with the barriers. Then, barriers were clustered per leg of journey (similar or identical barriers were merged), written on cards and pinned to a wall. Each participant got 20 adhesive dots to prioritise the clustered barriers per leg of journey 
by sticking as many adhesive dots as they want to the cards with the barriers they think are worst (Fig. 1b). Finally, this resulted in a list of prioritised barriers per leg of journey and user group. Focus groups took 2.5 hours with 6 to 8 attendees. Not all probing participants were involved in the subsequent prioritisation process. Nevertheless, all reprocessed diary entries were included as a basis for discussion within the focus groups. Finally, each participant got financial compensation and was handed a monthly ticket for public transportation.

\section{Results}

Quantitative analysis of the probes showed that number of identified barriers, as well as the average number of identified barriers per person was quite variable, especially between the groups of elderly and illiterates compared to the group of immigrants. Elderly persons and illiterates detected more different barriers than immigrants (Tab. 3). The participants roughly managed to take a picture for every diary entry they made. Summing up all three groups, in 5 cases they made two pictures depicting the same barrier and twice there was a diary entry without a picture.

The number of suggestions made for every detected barrier varied as well. Elderly gave suggestions for $73 \%$ of their total number of detected barriers and illiterates for $89 \%$. The immigrants suggested improvement for only $13 \%$ (Tab. 3).

Table 3. Number of detected barriers, diary entries, and observations per end user group

\begin{tabular}{lccc}
\hline & $\begin{array}{c}\text { Elderly } \\
(\mathrm{N}=9)\end{array}$ & $\begin{array}{c}\text { Illiterates } \\
(\mathrm{N}=11)\end{array}$ & $\begin{array}{c}\text { Immigrants } \\
(\mathrm{N}=7)\end{array}$ \\
\hline $\begin{array}{l}\text { number of different barriers } \\
\text { detected }\end{array}$ & 30 & 30 & 16 \\
$\begin{array}{l}\text { total number of diary entries } \\
\begin{array}{l}\text { Average number diary entries } \\
\text { per person }\end{array}\end{array}$ & 51 & 70 & 16 \\
$\begin{array}{l}\text { number of constructional and } \\
\begin{array}{l}\text { infrastructural barriers detected } \\
\text { number of psychosocial barriers }\end{array}\end{array}$ & 5.7 & 6.4 & 2.3 \\
$\begin{array}{l}\text { detected } \\
\text { number of suggestions for }\end{array}$ & 5 & 69 & 15 \\
$\begin{array}{l}\text { improvement } \\
\text { amount of observed behavioural } \\
\text { reactions in the surrounding }\end{array}$ & $24,(47.1 \%)$ & $29,(41.4 \%)$ & $1,(12.5 \%)$ \\
\hline
\end{tabular}

By means of the different type of used material, participants from all user groups were able to report constructional or infrastructural issues and limitations (e.g. step entry to tramway, no rain shield at the station). Participants mostly documented barriers, which referred to their lowered ability to perceive and understand information properly and immediately.

Interestingly, although the instructed goal of the study was to detect barriers, which realistically could be avoided by the use of a continuous mobile assistance (e.g. navigator app), also psycho-social issues (e.g. unfriendly bus drivers, crowding, smell 
disturbance) were noted into the diary. Especially elderly participants mentioned their perceptions in relation with social aspects and experienced discomfort (Tab. 3).

\section{Discussion and Conclusion}

According to the results we assume that the shaping of the packages for elderlies was well chosen for study purpose in general. Elderlies might need the possibility to reflect on additional impressions, even if they do not match the study focus, in order to keep them on track. However, due to the well-structured diary entries, it was easy to filter the non-focus entries out. Nevertheless, for further application of the DCPs, a refinement of the briefing, the checklist and a reminder task should be considered.

Overall, there were differences in the amount of detected barriers between the groups. Immigrants provided less data than the other groups. Although this user group was similarly instructed on the purpose of the study, they captured less barriers related to constructional and infrastructural issues, but also related to social interaction with others. Intercultural concerns appeared when husbands of participants forbid their wives to continue in the project. Although the procedural details were instructed in respect of any ethnic principles, people feared to violate their religious rules. Thus, in order to enhance compliance of immigrants, for further studies, we suggest to involve not only the participant himself/ herself to the study procedure, but also the surrounding family. As language barriers are persistent for immigrants, special translation and support efforts must be afforded for allowing immigrants to appropriately follow the instructions and discussions.

Although illiterates were impaired in writing and reading, they produced the largest number of diary entries in audio form. Most participants used the voice recorder without any problems, as well as they reported positive experience to document impressions without being constraint due to their minor writing skills. Another possible reason for this result is seen in the manipulation of the emphasis of verbal instruction and the related motivational effect. Illiterates were more motivated to solve the tasks if they were reinforced being 'researchers themselves, helping scientists to see the world with their eyes', and also having the possibility to improve life quality of others, especially their corresponding social group.

Gaver and colleagues [7] state that their 'results are impossible to analyse or even interpret clearly, because they reflect too many layers of influence and constraint'. In comparison, data from DCPs can be processed more easily, because of minor diversity in the data. However, every researcher should always reflect about the preferred kind of data required to follow research purpose. Accordingly, DCPs should be applied to projects or studies that are interested in a specific aspect of a target group and not in their whole everyday living. We assume that DCPs can be applied to serve as a substitute of traditional methods for requirement analysis (e.g. interviews, focus-groups). They are easily adaptable to the characteristics of the target group (e.g. verbal skills), as well as they provide data that can be processed quickly.

Generally, for the application of participatory methods, tools have to be adapted to the special needs of the potential 'researcher' carefully, otherwise relevant and important data might get lost. In contrast to common Cultural Probes, DCPs draw a less holistic picture of a users living context. The data is directed to the exact research 
question only and therefore provides more focused results. However, due to the preand post-data-collection contact with the user groups, it conveys a keen sense of user experience in a certain context. When Gaver and colleagues used domestic probe packages in 2004 [7], they used a purposely uncontrolled and uncontrollable approach. They provided participants with many diverse probing tools, such as a dream recorder or a friends and family map. It can be assumed that this method provides results of a wider and more global impression of how people live and how to design for them. Though, conducting Cultural Probes in terms of requirement analysis is a completely different approach. In order to extract concrete problems and user needs in a certain context, a more directed method is useful.

Altogether, we experienced the DCPs as a well performing method in terms of providing structured and pre-sorted data on the need of solutions for certain barriers. Although we do not want to claim about the novelty of our method too much, there is novelty concerning the application to this setting. The method generated useful data, which was processed in further tasks for the improvement of public transportation for people with special needs through a mobile assistance service. The raw data collection, the questions for the improvement of the barriers, the pre-interviews and subsequent focus groups including a prioritisation process, made the method expedient and valuable in terms of requirement analysis, which can be easily adapted to a different context.

\section{References}

1. Gaver, B., Dunne, T., Pacenti, E.: Design: Cultural Probes. Interactions 6(1), 21-29 (1999)

2. Palen, L., Saltzman, M.: Voice-Mail Diary Studies for Naturalistic Data Capture under Mobile Conditions. In: CSCW 2002: Proc. of the 2002 ACM Conference on Computer Supported Cooperative Work, pp. 87-95. ACM Press, New York (2002)

3. Hutchinson, H., Mackay, W., Westerlund, B., Benderson, B.B., Druin, A., Plaisant, C., Beaudouin-Lafon, M., Conversy, S., Evans, H., Hansen, H., Roussel, N., Eiderbäck, B.: Technology Probes: Inspiring Design for and with Families. In: Proc. CHI 2003, pp. 17-24. ACM Press, New York (2003)

4. Mättelmäki, T., Battarbee, K.: Empathy Probes. In: Proc. PDC 2002, pp. 266-271. CPSR (2002)

5. Hulkko, S., Mattelmäki, T., Virtanen, K., Keinonen, T.: Mobile Probes. In: Proc. NordiCHI 2004, pp. 43-51. ACM Press, Tampere (2004)

6. Crabtree, A., Hemmings, T., Rodden, T., Cheverst, K., Clarke, K., Dewsbury, G., Hughes, J., Rouncefield, M.: Designing With Care: Adapting Cultural Probes to Inform Design in Sensitive Settings. In: Proc. OzCHI 2004, pp. 4-13. Ergonomics Society of Australia, Brisbane (2004)

7. Gaver, W., Boucher, A., Pennington, S., Walker, B.: Cultural Probes and the Value of Uncertainty. Interactions 11(5), 53-56 (2004) 